Mathematical Modelling and AnAlysis

Volume 18 Number 3, June 2013, 309-324

http://dx.doi.org/10.3846/13926292.2013.804009

(c) Vilnius Gediminas Technical University, 2013
Publisher: Taylor\&Francis and VGTU

http://www.tandfonline.com/TMMA

Print ISSN: 1392-6292

Online ISSN: 1648-3510

\title{
Positive Solutions for Singular Systems of Higher-Order Multi-Point Boundary Value Problems
}

\author{
Johnny Henderson ${ }^{a}$ and Rodica Luca ${ }^{b}$ \\ ${ }^{a}$ Baylor University \\ 76798 Waco, USA \\ ${ }^{b}$ Gh. Asachi Technical University \\ 700506 Iasi, Romania \\ E-mail: Johnny_Henderson@baylor.edu \\ E-mail(corresp.): rluca@math.tuiasi.ro
}

Received June 27, 2012; revised April 10, 2013; published online June 1, 2013

Abstract. We investigate the existence of positive solutions for systems of singular nonlinear higher-order differential equations subject to multi-point boundary conditions.

Keywords: higher-order differential system, singular, multi-point boundary conditions, positive solutions.

AMS Subject Classification: 34B10; 34B18.

\section{Introduction}

We consider the singular system of nonlinear higher-order ordinary differential equations

$$
\begin{cases}u^{(n)}(t)+f(t, v(t))=0, & t \in(0, T), n \in \mathbb{N}, n \geq 2 \\ v^{(m)}(t)+g(t, u(t))=0, & t \in(0, T), m \in \mathbb{N}, m \geq 2\end{cases}
$$

with the multi-point boundary conditions

$$
\begin{cases}u(0)=u^{\prime}(0)=\cdots=u^{(n-2)}(0)=0, & u(T)=\sum_{i=1}^{p-2} a_{i} u\left(\xi_{i}\right), \quad p \in \mathbb{N}, p \geq 3, \\ v(0)=v^{\prime}(0)=\cdots=v^{(m-2)}(0)=0, & v(T)=\sum_{i=1}^{q-2} b_{i} v\left(\eta_{i}\right), \quad q \in \mathbb{N}, q \geq 3 .\end{cases}
$$

We present some weaker assumptions on $f$ and $g$, which do not possess any sublinear or superlinear growth conditions and may be singular at $t=0$ 
and/or $t=T$, such that positive solutions for problem $(S)-(B C)$ exist. By a positive solution of $(S)-(B C)$, we understand a pair of functions $(u, v) \in$ $\left(C\left([0, T] ; \mathbb{R}_{+}\right) \cap C^{n}((0, T))\right) \times\left(C\left([0, T] ; \mathbb{R}_{+}\right) \cap C^{m}((0, T))\right)$ satisfying $(S)$ and $(B C)$ with

$$
\sup _{t \in[0, T]} u(t)>0, \quad \sup _{t \in[0, T]} v(t)>0 .
$$

This problem is a generalization of the problem studied in [7], where $n=m=2$. In [12], the authors investigated the existence of positive solutions for system $(S)$ with $n=m=2$ and the boundary conditions

$$
u(0)=0, u(1)=\alpha u(\eta), v(0)=0, v(1)=\alpha v(\eta), \quad \eta \in(0,1), 0<\alpha \eta<1 .
$$

In [16], the authors studied the existence and multiplicity of positive solutions for system $(S)$ with $n=m=2, T=1$ and boundary conditions which contain only one intermediate point. We also mention the paper [14], where the authors used the fixed point index theory to prove the existence of positive solutions for the system $(S)$ with $f(t, v(t))$ and $g(t, u(t))$ replaced by $c(t) \widetilde{f}(u(t), v(t))$ and $d(t) \widetilde{g}(u(t), v(t))$, respectively, and $(B C)$, where $\frac{1}{2} \leq \xi_{1}<\xi_{2}<\cdots<\xi_{p-2}<$ $1, \frac{1}{2} \leq \eta_{1}<\eta_{2}<\cdots<\eta_{q-2}<1(T=1)$. Other systems with various nonlocal boundary conditions were investigated in the papers $[2,3,5,8,9,15]$. Some multi-point boundary value problems for systems of ordinary differential equations which involve positive eigenvalues were studied in recent years by using the Guo-Krasnosel'skii fixed point theorem. In [4], the authors give sufficient conditions for $\lambda, \mu, f$ and $g$ such that the system

$$
\left\{\begin{array}{l}
u^{(n)}(t)+\lambda c(t) f(u(t), v(t))=0, \quad t \in(0, T), n \in \mathbb{N}, n \geq 2, \\
v^{(m)}(t)+\mu d(t) g(u(t), v(t))=0, \quad t \in(0, T), m \in \mathbb{N}, m \geq 2,
\end{array}\right.
$$

with the boundary conditions $(B C)$ has positive solutions $(u(t) \geq 0, v(t) \geq 0$ for all $t \in[0, T]$ and $(u, v) \neq(0,0))$. The system $\left(S_{1}\right)$ with $n=m=2$ and the multi-point boundary conditions

$$
\begin{cases}\alpha u(0)-\beta u^{\prime}(0)=0, & u(T)=\sum_{i=1}^{p-2} a_{i} u\left(\xi_{i}\right), \quad p \in \mathbb{N}, p \geq 3, \\ \gamma v(0)-\delta v^{\prime}(0)=0, & v(T)=\sum_{i=1}^{q-2} b_{i} v\left(\eta_{i}\right), \quad q \in \mathbb{N}, \quad q \geq 3,\end{cases}
$$

has been investigated in [6].

In recent years, multi-point boundary value problems for second-order or higher-order differential or difference equations/systems have been investigated by many authors, by using different methods such as fixed point theorems in cones, the Leray-Schauder continuation theorem and its nonlinear alternatives, and the coincidence degree theory.

In Section 2, we shall present some auxiliary results which investigate two boundary value problems for higher-order equations (the problems (2.1)-(2.2) and (2.4)-(2.5) below). In Section 3, we shall prove two existence results for the positive solutions with respect to a cone for our problem $(S)-(B C)$, which are based on the Guo-Krasnosel'skii fixed point theorem, presented below. 
Theorem 1. Let $X$ be a Banach space and let $C \subset X$ be a cone in $X$. Assume $\Omega_{1}$ and $\Omega_{2}$ are bounded open subsets of $X$ with $0 \in \Omega_{1} \subset \overline{\Omega_{1}} \subset \Omega_{2}$ and let $\mathcal{A}: C \cap\left(\overline{\Omega_{2}} \backslash \Omega_{1}\right) \rightarrow C$ be a completely continuous operator such that, either

i) $\|\mathcal{A} u\| \leq\|u\|, u \in C \cap \partial \Omega_{1}$, and $\|\mathcal{A} u\| \geq\|u\|, u \in C \cap \partial \Omega_{2}$, or

ii) $\|\mathcal{A} u\| \geq\|u\|, u \in C \cap \partial \Omega_{1}$, and $\|\mathcal{A} u\| \leq\|u\|, u \in C \cap \partial \Omega_{2}$.

Then $\mathcal{A}$ has a fixed point in $C \cap\left(\overline{\Omega_{2}} \backslash \Omega_{1}\right)$.

Finally, in Section 4, we shall present some examples which illustrate our main results.

\section{Auxiliary Results}

In this section, we shall present some auxiliary results from [10,11] (see also [13]) related to the following $n$ th-order differential equation with $p$-point boundary conditions

$$
\begin{aligned}
& u^{(n)}(t)+y(t)=0, \quad t \in(0, T), \\
& u(0)=u^{\prime}(0)=\cdots=u^{(n-2)}(0)=0, \quad u(T)=\sum_{i=1}^{p-2} a_{i} u\left(\xi_{i}\right) .
\end{aligned}
$$

Lemma 1. If $d=T^{n-1}-\sum_{i=1}^{p-2} a_{i} \xi_{i}^{n-1} \neq 0,0<\xi_{1}<\cdots<\xi_{p-2}<T$ and $y \in C([0, T])$, then the solution of $(2.1)-(2.2)$ is given by

$$
\begin{aligned}
u(t)= & \frac{t^{n-1}}{d(n-1) !} \int_{0}^{T}(T-s)^{n-1} y(s) d s-\frac{t^{n-1}}{d(n-1) !} \sum_{i=1}^{p-2} a_{i} \int_{0}^{\xi_{i}}\left(\xi_{i}-s\right)^{n-1} y(s) d s \\
& -\frac{1}{(n-1) !} \int_{0}^{t}(t-s)^{n-1} y(s) d s, \quad 0 \leq t \leq T .
\end{aligned}
$$

Lemma 2. Under the assumptions of Lemma 1, the Green's function for the boundary value problem (2.1)-(2.2) is given by

$$
G_{1}(t, s)=\left\{\begin{array}{l}
\frac{t^{n-1}}{d(n-1) !}\left[(T-s)^{n-1}-\sum_{i=j+1}^{p-2} a_{i}\left(\xi_{i}-s\right)^{n-1}\right]-\frac{1}{(n-1) !}(t-s)^{n-1}, \\
\quad \text { if } \xi_{j} \leq s<\xi_{j+1}, \quad s \leq t, \\
\frac{t^{n-1}}{d(n-1) !}\left[(T-s)^{n-1}-\sum_{i=j+1}^{p-2} a_{i}\left(\xi_{i}-s\right)^{n-1}\right], \\
\quad \text { if } \xi_{j} \leq s<\xi_{j+1}, \quad s \geq t, j=0, \ldots, p-3, \\
\frac{t^{n-1}}{d(n-1) !}(T-s)^{n-1}-\frac{1}{(n-1) !}(t-s)^{n-1}, \quad \text { if } \xi_{p-2} \leq s \leq T, s \leq t, \\
\frac{t^{n-1}}{d(n-1) !}(T-s)^{n-1}, \quad \text { if } \xi_{p-2} \leq s \leq T, s \geq t\left(\xi_{0}=0\right),
\end{array}\right.
$$

for all $(t, s) \in[0, T] \times[0, T]$. 
Using the Heaviside function on $\mathbb{R}, H(x)=1$ for $x \geq 0$, and $H(x)=0$ for $x<0$, the above Green's function can be written in a compact form

$$
\begin{aligned}
G_{1}(t, s)= & \frac{t^{n-1}}{d(n-1) !}\left[(T-s)^{n-1}-\sum_{i=1}^{p-2} a_{i}\left(\xi_{i}-s\right)^{n-1} H\left(\xi_{i}-s\right)\right] \\
& -\frac{1}{(n-1) !}(t-s)^{n-1} H(t-s), \quad(t, s) \in[0, T] \times[0, T] .
\end{aligned}
$$

By using the above Green's function the solution of problem (2.1)-(2.2) is expressed as $u(t)=\int_{0}^{T} G_{1}(t, s) y(s) d s$.

Lemma 3. Under the assumptions of Lemma 1, the Green's function for the boundary value problem (2.1)-(2.2) can be expressed as

$$
G_{1}(t, s)=g_{1}(t, s)+\frac{t^{n-1}}{d} \sum_{i=1}^{p-2} a_{i} g_{1}\left(\xi_{i}, s\right)
$$

where

$$
g_{1}(t, s)=\frac{1}{(n-1) ! T^{n-1}}\left\{\begin{array}{l}
t^{n-1}(T-s)^{n-1}-T^{n-1}(t-s)^{n-1}, \quad 0 \leq s \leq t \leq T \\
t^{n-1}(T-s)^{n-1}, \quad 0 \leq t \leq s \leq T
\end{array}\right.
$$

Lemma 4. The function $g_{1}$ given in (2.3) has the properties:

a) $g_{1}:[0, T] \times[0, T] \rightarrow \mathbb{R}_{+}$is a continuous function and $g_{1}(t, s) \geq 0$ for all $(t, s) \in[0, T] \times[0, T]$.

b) $g_{1}(t, s) \leq g_{1}\left(\theta_{1}(s), s\right)$, for all $(t, s) \in[0, T] \times[0, T]$.

c) For any $c \in\left(0, \frac{T}{2}\right), \min _{t \in[c, T-c]} g_{1}(t, s) \geq \frac{c^{n-1}}{T^{n-1}} g_{1}\left(\theta_{1}(s), s\right)$, for all $s \in$ $[0, T]$

where $\theta_{1}(s)=s$ if $n=2$ and

$$
\theta_{1}(s)=\left\{\begin{array}{l}
\frac{s}{1-\left(1-\frac{s}{T}\right)^{\frac{n-1}{n-2}}}, \quad s \in(0, T], \\
\frac{T(n-2)}{n-1}, \quad s=0
\end{array} \quad \text { if } n \geq 3\right.
$$

Lemma 5. Assume that $d>0,0<\xi_{1}<\cdots<\xi_{p-2}<T, a_{i} \geq 0$ for all $i=1, \ldots, p-2$. Then the Green's function $G_{1}$ of the problem (2.1)-(2.2) is continuous on $[0, T] \times[0, T]$ and satisfies $G_{1}(t, s) \geq 0$ for all $(t, s) \in[0, T] \times$ $[0, T]$. Moreover, if $y \in C([0, T])$ satisfies $y(t) \geq 0$ for all $t \in[0, T]$, then the unique solution $u$ of problem $(2.1)-(2.2)$ satisfies $u(t) \geq 0$ for all $t \in[0, T]$.

Lemma 6. Assume that $d>0,0<\xi_{1}<\cdots<\xi_{p-2}<T, a_{i} \geq 0$ for all $i=1, \ldots, p-2$. Then the Green's function $G_{1}$ of problem (2.1)-(2.2) satisfies the inequalities 
a) $G_{1}(t, s) \leq I_{1}(s), \forall(t, s) \in[0, T] \times[0, T]$, where

$$
I_{1}(s)=g_{1}\left(\theta_{1}(s), s\right)+\frac{T^{n-1}}{d} \sum_{i=1}^{p-2} a_{i} g_{1}\left(\xi_{i}, s\right), \quad \forall s \in[0, T]
$$

b) For every $c \in\left(0, \frac{T}{2}\right)$,

$$
\min _{t \in[c, T-c]} G_{1}(t, s) \geq \frac{c^{n-1}}{T^{n-1}} I_{1}(s), \quad \forall s \in[0, T] .
$$

Lemma 7. Assume that $d>0,0<\xi_{1}<\cdots<\xi_{p-2}<T, a_{i} \geq 0$ for all $i=1, \ldots, p-2, c \in\left(0, \frac{T}{2}\right)$ and $y \in C([0, T])$ satisfies $y(t) \geq 0$ for all $t \in[0, T]$. Then the solution $u(t), t \in[0, T]$, of problem (2.1)-(2.2) satisfies the inequality

$$
\min _{t \in[c, T-c]} u(t) \geq \frac{c^{n-1}}{T^{n-1}} \max _{t^{\prime} \in[0, T]} u\left(t^{\prime}\right) .
$$

We can also formulate similar results as Lemmas 1-7 above for the boundary value problem

$$
\begin{gathered}
v^{(m)}(t)+h(t)=0, \quad t \in(0, T) \\
v(0)=v^{\prime}(0)=\cdots=v^{(m-2)}(0)=0, \quad v(T)=\sum_{i=1}^{q-2} b_{i} v\left(\eta_{i}\right),
\end{gathered}
$$

where $0<\eta_{1}<\cdots<\eta_{q-2}<T, b_{i} \geq 0$ for $i=1, \ldots, q-2$, and $h \in C([0, T])$. If $e=T^{m-1}-\sum_{i=1}^{q-2} b_{i} \eta_{i}^{m-1} \neq 0$, we denote by $G_{2}$ the Green's function associated to problem (2.4)-(2.5) and defined in a similar manner as $G_{1}$. We also denote by $g_{2}, \theta_{2}$ and $I_{2}$ the corresponding functions for (2.4)-(2.5) defined in a similar manner as $g_{1}, \theta_{1}$ and $I_{1}$, respectively.

\section{Main Results}

In this section, we shall investigate the existence of positive solutions for our problem $(S)-(B C)$, under various assumptions on singular functions $f$ and $g$.

We present the assumptions that we shall use in the sequel.

(H1) $0<\xi_{1}<\cdots<\xi_{p-2}<T, a_{i} \geq 0, i=1, \ldots, p-2, d=T^{n-1}-$ $\sum_{i=1}^{p-2} a_{i} \xi_{i}^{n-1}>0,0<\eta_{1}<\cdots<\eta_{q-2}<T, b_{i} \geq 0, i=1, \ldots, q-2$, $e=T^{m-1}-\sum_{i=1}^{q-2} b_{i} \eta_{i}^{m-1}>0$.

(H2) Functions $f, g \in C\left((0, T) \times \mathbb{R}_{+}, \mathbb{R}_{+}\right)$and there exist $p_{i} \in C\left((0, T), \mathbb{R}_{+}\right)$, $q_{i} \in C\left(\mathbb{R}_{+}, \mathbb{R}_{+}\right), i=1,2$, with $0<\int_{0}^{T} p_{i}(t) d t<\infty, i=1,2, q_{1}(0)=0$, $q_{2}(0)=0$ such that

$$
f(t, x) \leq p_{1}(t) q_{1}(x), \quad g(t, x) \leq p_{2}(t) q_{2}(x), \quad \forall t \in(0, T), x \in \mathbb{R}_{+} .
$$


(H3) There exist $r_{1}, r_{2} \in(0, \infty)$ with $r_{1} r_{2} \geq 1$ such that

$$
\text { i) } q_{10}^{s}=\limsup _{x \rightarrow 0^{+}} \frac{q_{1}(x)}{x^{r_{1}}} \in[0, \infty) ; \quad \text { ii) } \quad q_{20}^{s}=\limsup _{x \rightarrow 0^{+}} \frac{q_{2}(x)}{x^{r_{2}}}=0 .
$$

(H4) There exist $l_{1}, l_{2} \in(0, \infty)$ with $l_{1} l_{2} \geq 1$ and $c \in\left(0, \frac{T}{2}\right)$ such that

$$
\begin{aligned}
\text { i) } f_{\infty}^{i} & =\liminf _{x \rightarrow \infty} \inf _{t \in[c, T-c]} \frac{f(t, x)}{x^{l_{1}}} \in(0, \infty] ; \\
\text { ii) } g_{\infty}^{i} & =\liminf _{x \rightarrow \infty} \inf _{t \in[c, T-c]} \frac{g(t, x)}{x^{l_{2}}}=\infty .
\end{aligned}
$$

(H5) There exist $\alpha_{1}, \alpha_{2} \in(0, \infty)$ with $\alpha_{1} \alpha_{2} \leq 1$ such that

$$
\text { i) } \quad q_{1 \infty}^{s}=\limsup _{x \rightarrow \infty} \frac{q_{1}(x)}{x^{\alpha_{1}}} \in[0, \infty) ; \quad \text { ii) } \quad q_{2 \infty}^{s}=\limsup _{x \rightarrow \infty} \frac{q_{2}(x)}{x^{\alpha_{2}}}=0 .
$$

(H6) There exist $\beta_{1}, \beta_{2} \in(0, \infty)$ with $\beta_{1} \beta_{2} \leq 1$ and $c \in\left(0, \frac{T}{2}\right)$ such that

$$
\begin{aligned}
& \text { i) } f_{0}^{i}=\liminf _{x \rightarrow 0^{+}} \inf _{t \in[c, T-c]} \frac{f(t, x)}{x^{\beta_{1}}} \in(0, \infty] ; \\
& \text { ii) } g_{0}^{i}=\liminf _{x \rightarrow 0^{+}} \inf _{t \in[c, T-c]} \frac{g(t, x)}{x^{\beta_{2}}}=\infty .
\end{aligned}
$$

The pair of functions $(u, v) \in\left(C([0, T]) \cap C^{n}((0, T))\right) \times(C([0, T]) \cap$ $\left.C^{m}((0, T))\right)$ is a solution for our problem $(S)-(B C)$ if and only if $(u, v) \in$ $C([0, T]) \times C([0, T])$ is a solution for the nonlinear integral equations

$$
\begin{cases}u(t)=\int_{0}^{T} G_{1}(t, s) f(s, v(s)) d s, & t \in[0, T] \\ v(t)=\int_{0}^{T} G_{2}(t, s) g(s, u(s)) d s, & t \in[0, T] .\end{cases}
$$

The system (3.1) can be written as the nonlinear integral system

$$
\left\{\begin{array}{l}
u(t)=\int_{0}^{T} G_{1}(t, s) f\left(s, \int_{0}^{T} G_{2}(s, \tau) g(\tau, u(\tau)) d \tau\right) d s, \quad t \in[0, T] \\
v(t)=\int_{0}^{T} G_{2}(t, s) g(s, u(s)) d s, \quad t \in[0, T]
\end{array}\right.
$$

We consider the Banach space $X=C([0, T])$ with supremum norm $\|u\|=$ $\sup _{t \in[0, T]}|u(t)|$ and define the cone $P \subset X$ by $P=\{u \in X, u(t) \geq 0, \forall t \in$ $[0, T]\}$. For any $r>0$, let

$$
B_{r}=\{u \in C([0, T]),\|u\|<r\}, \quad \partial B_{r}=\{u \in C([0, T]),\|u\|=r\} .
$$

We also define the operator $\mathcal{A}: P \rightarrow X$ by

$$
\mathcal{A}(u)(t)=\int_{0}^{T} G_{1}(t, s) f\left(s, \int_{0}^{T} G_{2}(s, \tau) g(\tau, u(\tau)) d \tau\right) d s .
$$


Lemma 8. Assume that $(H 1)-(H 2)$ hold. Then $\mathcal{A}: P \rightarrow P$ is completely continuous.

Proof. We denote $\alpha=\int_{0}^{T} I_{1}(s) p_{1}(s) d s$ and $\beta=\int_{0}^{T} I_{2}(s) p_{2}(s) d s$. Using (H2), we deduce that $0<\alpha<\infty$ and $0<\beta<\infty$. By Lemma 5 and the corresponding lemma for $G_{2}$, we get that $\mathcal{A}$ maps $P$ into $P$.

We shall prove that $\mathcal{A}$ maps bounded sets into relatively compact sets. Suppose $D \subset P$ is an arbitrary bounded set. First we prove that $\mathcal{A}(D)$ is a bounded set. Because $D$ is bounded, then there exists $M_{1}>0$ such that $\|u\| \leq M_{1}$ for all $u \in D$. By the continuity of $q_{2}$, there exists $M_{2}>0$ such that $M_{2}=\sup _{x \in\left[0, M_{1}\right]} q_{2}(x)$. By using Lemma 6 for $G_{2}$, for any $u \in D$ and $s \in[0, T]$, we obtain

$$
\begin{aligned}
\int_{0}^{T} G_{2}(s, \tau) g(\tau, u(\tau)) d \tau & \leq \int_{0}^{T} G_{2}(s, \tau) p_{2}(\tau) q_{2}(u(\tau)) d \tau \\
& \leq M_{2} \int_{0}^{T} I_{2}(\tau) p_{2}(\tau) d \tau=\beta M_{2}
\end{aligned}
$$

Because $q_{1}$ is a continuous function, there exists $M_{3}>0$ such that $M_{3}=$ $\sup _{x \in\left[0, \beta M_{2}\right]} q_{1}(x)$. Therefore, from (3.2), (H2) and Lemma 6, we deduce

$$
\begin{aligned}
(\mathcal{A} u)(t) & =\int_{0}^{T} G_{1}(t, s) f\left(s, \int_{0}^{T} G_{2}(s, \tau) g(\tau, u(\tau)) d \tau\right) d s \\
& \leq \int_{0}^{T} G_{1}(t, s) p_{1}(s) q_{1}\left(\int_{0}^{T} G_{2}(s, \tau) g(\tau, u(\tau)) d \tau\right) d s \\
& \leq M_{3} \int_{0}^{T} I_{1}(s) p_{1}(s) d s=\alpha M_{3}, \quad \forall t \in[0, T] .
\end{aligned}
$$

So, $\|\mathcal{A} u\| \leq \alpha M_{3}$ for all $u \in D$. Therefore $\mathcal{A}(D)$ is bounded.

In what follows, we shall prove that $\mathcal{A}(D)$ is equicontinuous. By using Lemma 3, we have

$$
\begin{aligned}
(\mathcal{A} u)(t)= & \int_{0}^{T} G_{1}(t, s) f\left(s, \int_{0}^{T} G_{2}(s, \tau) g(\tau, u(\tau)) d \tau\right) d s \\
= & \int_{0}^{T}\left[g_{1}(t, s)+\frac{t^{n-1}}{d} \sum_{i=1}^{p-2} a_{i} g_{1}\left(\xi_{i}, s\right)\right] f\left(s, \int_{0}^{T} G_{2}(s, \tau) g(\tau, u(\tau)) d \tau\right) d s \\
= & \frac{1}{(n-1) ! T^{n-1}} \int_{0}^{t}\left[t^{n-1}(T-s)^{n-1}-T^{n-1}(t-s)^{n-1}\right] \\
& \times f\left(s, \int_{0}^{T} G_{2}(s, \tau) g(\tau, u(\tau)) d \tau\right) d s \\
& +\frac{1}{(n-1) ! T^{n-1}} \int_{t}^{T} t^{n-1}(T-s)^{n-1} f\left(s, \int_{0}^{T} G_{2}(s, \tau) g(\tau, u(\tau)) d \tau\right) d s \\
& +\frac{t^{n-1}}{d} \sum_{i=1}^{p-2} a_{i} \int_{0}^{T} g_{1}\left(\xi_{i}, s\right) f\left(s, \int_{0}^{T} G_{2}(s, \tau) g(\tau, u(\tau)) d \tau\right) d s, \quad \forall t \in[0, T]
\end{aligned}
$$


Therefore, for any $t \in(0, T)$, we obtain

$$
\begin{aligned}
(\mathcal{A} u)^{\prime}(t)= & \frac{t^{n-1}(T-t)^{n-1}-T^{n-1}(t-t)^{n-1}}{(n-1) ! T^{n-1}} f\left(t, \int_{0}^{T} G_{2}(t, \tau) g(\tau, u(\tau)) d \tau\right) \\
& +\int_{0}^{t} \frac{(n-1) t^{n-2}(T-s)^{n-1}-(n-1) T^{n-1}(t-s)^{n-2}}{(n-1) ! T^{n-1}} \\
& \times f\left(s, \int_{0}^{T} G_{2}(s, \tau) g(\tau, u(\tau)) d \tau\right) d s \\
& -\frac{t^{n-1}(T-t)^{n-1}}{(n-1) ! T^{n-1}} f\left(t, \int_{0}^{T} G_{2}(t, \tau) g(\tau, u(\tau)) d \tau\right) \\
& +\int_{t}^{T} \frac{(n-1) t^{n-2}(T-s)^{n-1}}{(n-1) ! T^{n-1}} f\left(s, \int_{0}^{T} G_{2}(s, \tau) g(\tau, u(\tau)) d \tau\right) \\
& +\frac{(n-1) t^{n-2} \sum_{i=1}^{p-2} a_{i} \int_{0}^{T} g_{1}\left(\xi_{i}, s\right) f\left(s, \int_{0}^{T} G_{2}(s, \tau) g(\tau, u(\tau)) d \tau\right) d s .}{d}
\end{aligned}
$$

So, for any $t \in(0, T)$, we deduce

$$
\begin{aligned}
\left|(\mathcal{A} u)^{\prime}(t)\right| & \leq \int_{0}^{t} \frac{t^{n-2}(T-s)^{n-1}+T^{n-1}(t-s)^{n-2}}{(n-2) ! T^{n-1}} p_{1}(s) \\
& \times g_{1}\left(\int_{0}^{T} G_{2}(s, \tau) g(\tau, u(\tau)) d \tau\right) d s \\
& +\int_{t}^{T} \frac{t^{n-2}(T-s)^{n-1}}{(n-2) ! T^{n-1}} p_{1}(s) q_{1}\left(\int_{0}^{T} G_{2}(s, \tau) g(\tau, u(\tau)) d \tau\right) d s \\
& +\frac{(n-1) t^{n-2} \sum_{i=1}^{p-2} a_{i} \int_{0}^{T} g_{1}\left(\xi_{i}, s\right) p_{1}(s) q_{1}\left(\int_{0}^{T} G_{2}(s, \tau) g(\tau, u(\tau)) d \tau\right) d s}{d} \\
& \leq M_{3}\left(\int_{0}^{t} \frac{t^{n-2}(T-s)^{n-1}+T^{n-1}(t-s)^{n-2}}{(n-2) ! T^{n-1}} p_{1}(s) d s\right. \\
& +\int_{t}^{T} \frac{t^{n-2}(T-s)^{n-1}}{(n-2) ! T^{n-1}} p_{1}(s) d s \\
& +\frac{\left.(n-1) t^{n-2} \sum_{i=1}^{p-2} a_{i} \int_{0}^{T} g_{1}\left(\xi_{i}, s\right) p_{1}(s) d s\right) .}{d}
\end{aligned}
$$

We denote

$$
\begin{aligned}
h(t)= & \int_{0}^{t} \frac{t^{n-2}(T-s)^{n-1}+T^{n-1}(t-s)^{n-2}}{(n-2) ! T^{n-1}} p_{1}(s) d s \\
& +\int_{t}^{T} \frac{t^{n-2}(T-s)^{n-1}}{(n-2) ! T^{n-1}} p_{1}(s) d s, \\
\mu(t)= & h(t)+\frac{(n-1) t^{n-2}}{d} \sum_{i=1}^{p-2} a_{i} \int_{0}^{T} g_{1}\left(\xi_{i}, s\right) p_{1}(s) d s, \quad t \in(0, T) .
\end{aligned}
$$


For the integral of the function $h$, by exchanging the order of integration, we obtain

$$
\begin{aligned}
& \int_{0}^{T} h(t) d t=\int_{0}^{T}\left(\int_{0}^{t} \frac{t^{n-2}(T-s)^{n-1}+T^{n-1}(t-s)^{n-2}}{(n-2) ! T^{n-1}} p_{1}(s) d s\right) d t \\
& +\int_{0}^{T}\left(\int_{t}^{T} \frac{t^{n-2}(T-s)^{n-1}}{(n-2) ! T^{n-1}} p_{1}(s) d s\right) d t \\
& =\int_{0}^{T}\left(\int_{s}^{T} \frac{t^{n-2}(T-s)^{n-1}+T^{n-1}(t-s)^{n-2}}{(n-2) ! T^{n-1}} p_{1}(s) d t\right) d s \\
& +\int_{0}^{T}\left(\int_{0}^{s} \frac{t^{n-2}(T-s)^{n-1}}{(n-2) ! T^{n-1}} p_{1}(s) d t\right) d s \\
& =\int_{0}^{T} \frac{(T-s)^{n-1}}{(n-2) ! T^{n-1}} p_{1}(s)\left(\int_{s}^{T} t^{n-2} d t\right) d s+\int_{0}^{T} \frac{p_{1}(s)}{(n-2) !}\left(\int_{s}^{T}(t-s)^{n-2} d t\right) d s \\
& +\int_{0}^{T} \frac{(T-s)^{n-1}}{(n-2) ! T^{n-1}} p_{1}(s)\left(\int_{0}^{s} t^{n-2} d t\right) d s \\
& =\int_{0}^{T} \frac{(T-s)^{n-1}}{(n-2) ! T^{n-1}}\left(\frac{T^{n-1}-s^{n-1}}{n-1}\right) p_{1}(s) d s+\int_{0}^{T} \frac{p_{1}(s)(T-s)^{n-1}}{(n-1) !} d s \\
& +\int_{0}^{T} \frac{(T-s)^{n-1} s^{n-1}}{(n-1) ! T^{n-1}} p_{1}(s) d s \\
& =\frac{2}{(n-1) !} \int_{0}^{T}(T-s)^{n-1} p_{1}(s) d s<\infty .
\end{aligned}
$$

For the integral of the function $\mu$, we have

$$
\begin{aligned}
& \int_{0}^{T} \mu(t) d t=\int_{0}^{T} h(t) d t+\frac{n-1}{d} \sum_{i=1}^{p-2} a_{i} \int_{0}^{T} t^{n-2}\left(\int_{0}^{T} g_{1}\left(\xi_{i}, s\right) p_{1}(s) d s\right) d t \\
& \quad \leq \frac{2}{(n-1) !} \int_{0}^{T}(T-s)^{n-1} p_{1}(s) d s+\frac{T^{n-1}}{d} \sum_{i=1}^{p-2} a_{i} \int_{0}^{T} g_{1}\left(\theta_{1}(s), s\right) p_{1}(s) d s \\
& \quad \leq \frac{1}{(n-1) !}\left(2+\frac{T^{n-1}}{d} \sum_{i=1}^{p-2} a_{i}\right) \int_{0}^{T}(T-s)^{n-1} p_{1}(s) d s<\infty
\end{aligned}
$$

We deduce that $\mu \in L^{1}(0, T)$. Thus for any given $t_{1}, t_{2} \in[0,1]$ with $t_{1} \leq t_{2}$ and $u \in D$, by (3.4), we obtain

$$
\left|(\mathcal{A} u)\left(t_{1}\right)-(\mathcal{A} u)\left(t_{2}\right)\right|=\left|\int_{t_{1}}^{t_{2}}(\mathcal{A} u)^{\prime}(t) d t\right| \leq M_{3} \int_{t_{1}}^{t_{2}} \mu(t) d t .
$$

From (3.5), (3.6) and absolute continuity of the integral function, we obtain that $\mathcal{A}(D)$ is equicontinuous. This conclusion together with (3.3) and Ascoli-Arzelà theorem yields that $\mathcal{A}(D)$ is relatively compact. Therefore $\mathcal{A}$ is a compact operator.

By using similar arguments as those used in the proof of Lemma 2.4 from [12], we can show that $\mathcal{A}$ is continuous on $P$. Therefore $\mathcal{A}: P \rightarrow P$ is completely continuous. 
For $c \in(0, T / 2)$, we define the cone

$$
P_{0}=\left\{u \in X, u(t) \geq 0, \forall t \in[0, T], \min _{t \in[c, T-c]} u(t) \geq \gamma\|u\|\right\},
$$

where $\gamma=\min \left\{c^{n-1} / T^{n-1}, c^{m-1} / T^{m-1}\right\}$. Under assumptions $(H 1),(H 2)$, we have $\mathcal{A}(P) \subset P_{0}$. Indeed, for $u \in P$, let $v=\mathcal{A}(u)$. By Lemma 7 , we have

$$
\min _{t \in[c, T-c]} v(t) \geq \frac{c^{n-1}}{T^{n-1}}\|v\| \geq \gamma\|v\|
$$

that is $v \in P_{0}$.

Theorem 2. Assume that $(H 1)-(H 4)$ hold. Then the problem $(S)-(B C)$ has at least one positive solution $(u(t), v(t)), t \in[0, T]$.

Proof. We consider the cone $P_{0}$ with $c$ given in (H4). From (H3) i) and (H2), we deduce that there exists $C_{1}>0$ such that

$$
q_{1}(x) \leq C_{1} x^{r_{1}}, \quad \forall x \in[0,1] .
$$

From $(H 3)$ ii) and $(H 2)$, for $C_{2}=\min \left\{\left(1 /\left(C_{1} \alpha \beta^{r_{1}}\right)\right)^{1 / r_{1}}, 1 / \beta\right\}>0$, we deduce that there exists $\delta_{1} \in(0,1)$ such that

$$
q_{2}(x) \leq C_{2} x^{r_{2}}, \quad \forall x \in\left[0, \delta_{1}\right] .
$$

From (3.8), (H2) and Lemma 6 , for any $u \in \partial B_{\delta_{1}} \cap P_{0}$ and $s \in[0, T]$, we obtain

$$
\begin{aligned}
& \int_{0}^{T} G_{2}(s, \tau) g(\tau, u(\tau)) d \tau \leq \int_{0}^{T} G_{2}(s, \tau) p_{2}(\tau) q_{2}(u(\tau)) d \tau \\
& \quad \leq C_{2} \int_{0}^{T} G_{2}(s, \tau) p_{2}(\tau)(u(\tau))^{r_{2}} d \tau \\
& \quad \leq C_{2} \int_{0}^{T} I_{2}(\tau) p_{2}(\tau) d \tau \cdot\|u\|^{r_{2}}=C_{2} \beta \delta_{1}^{r_{2}} \leq \delta_{1}^{r_{2}}<1
\end{aligned}
$$

By using (3.7)-(3.9) and (H2), for any $u \in \partial B_{\delta_{1}} \cap P_{0}$ and $t \in[0, T]$, we obtain

$$
\begin{aligned}
(\mathcal{A} u)(t) & \leq \int_{0}^{T} G_{1}(t, s) p_{1}(s) q_{1}\left(\int_{0}^{T} G_{2}(s, \tau) g(\tau, u(\tau)) d \tau\right) d s \\
& \leq C_{1} \int_{0}^{T} G_{1}(t, s) p_{1}(s)\left(\int_{0}^{T} G_{2}(s, \tau) g(\tau, u(\tau)) d \tau\right)^{r_{1}} d s \\
& \leq C_{1} \int_{0}^{T} G_{1}(t, s) p_{1}(s)\left(\int_{0}^{T} G_{2}(s, \tau) p_{2}(\tau) q_{2}(u(\tau)) d \tau\right)^{r_{1}} d s \\
& \leq C_{1} \int_{0}^{T} G_{1}(t, s) p_{1}(s)\left(C_{2} \int_{0}^{T} G_{2}(s, \tau) p_{2}(\tau)(u(\tau))^{r_{2}} d \tau\right)^{r_{1}} d s \\
& \leq C_{1} \int_{0}^{T} I_{1}(s) p_{1}(s)\left(C_{2} \int_{0}^{T} I_{2}(\tau) p_{2}(\tau)(u(\tau))^{r_{2}} d \tau\right)^{r_{1}} d s
\end{aligned}
$$




$$
\begin{aligned}
& \leq C_{1} \int_{0}^{T} I_{1}(s) p_{1}(s) d s \cdot\left(C_{2} \int_{0}^{T} I_{2}(\tau) p_{2}(\tau) d \tau\right)^{r_{1}} \cdot\|u\|^{r_{1} r_{2}} \\
& \leq C_{1} \alpha\left(C_{2} \beta\right)^{r_{1}}\|u\|^{r_{1} r_{2}}=C_{1} C_{2}^{r_{1}} \alpha \beta^{r_{1}}\|u\|^{r_{1} r_{2}} \leq\|u\|^{r_{1} r_{2}} \leq\|u\| .
\end{aligned}
$$

Therefore

$$
\|\mathcal{A} u\| \leq\|u\|, \quad \forall u \in \partial B_{\delta_{1}} \cap P_{0} .
$$

From (H4) i), we deduce that there exist $C_{3}>0$ and $x_{1}>0$ such that

$$
f(t, x) \geq C_{3} x^{l_{1}}, \quad \forall x \geq x_{1}, \forall t \in[c, T-c] .
$$

We consider now

$$
C_{4}=\max \left\{\frac{T^{m-1}}{c^{m-1} \gamma^{l_{2}} \theta_{2}},\left(\frac{T^{l_{1}(m-1)+n-1}}{c^{l_{1}(m-1)+n-1} C_{3} \gamma^{l_{1} l_{2}} \theta_{1} \theta_{2}^{l_{1}}}\right)^{1 / l_{1}}\right\}>0,
$$

where $\theta_{1}=\int_{c}^{T-c} I_{1}(s) d s>0$ and $\theta_{2}=\int_{c}^{T-c} I_{2}(s) d s>0$. From (H4) ii), we deduce that there exists $x_{2} \geq 1$ such that

$$
g(t, x) \geq C_{4} x^{l_{2}}, \quad \forall x \geq x_{2}, \forall t \in[c, T-c] .
$$

Now we choose $R_{0}=\max \left\{x_{1}, x_{2}\right\}$ and $R>\max \left\{R_{0} / \gamma, R_{0}^{1 / l_{2}}\right\}$. Then for any $u \in \partial B_{R} \cap P_{0}$, we have $\min _{t \in[c, T-c]} u(t) \geq \gamma\|u\|=\gamma R>R_{0}$. By using (3.11) and (3.12), for any $u \in \partial B_{R} \cap P_{0}$ and $s \in[c, T-c]$, we obtain

$$
\begin{aligned}
\int_{0}^{T} & G_{2}(s, \tau) g(\tau, u(\tau)) d \tau \geq \int_{c}^{T-c} G_{2}(s, \tau) g(\tau, u(\tau)) d \tau \\
& \geq \frac{c^{m-1}}{T^{m-1}} \int_{c}^{T-c} I_{2}(\tau) C_{4}(u(\tau))^{l_{2}} d \tau \geq \frac{c^{m-1}}{T^{m-1}} C_{4} \gamma^{l_{2}} \int_{c}^{T-c} I_{2}(\tau) d \tau \cdot\|u\|^{l_{2}} \\
& \geq\|u\|^{l_{2}}=R^{l_{2}}>R_{0}
\end{aligned}
$$

Then for any $u \in \partial B_{R} \cap P_{0}$ and $t \in[c, T-c]$, we have

$$
\begin{aligned}
(\mathcal{A} u)(t) & =\int_{0}^{T} G_{1}(t, s) f\left(s, \int_{0}^{T} G_{2}(s, \tau) g(\tau, u(\tau)) d \tau\right) d s \\
& \geq \int_{c}^{T-c} G_{1}(t, s) f\left(s, \int_{0}^{T} G_{2}(s, \tau) g(\tau, u(\tau)) d \tau\right) d s \\
& \geq C_{3} \int_{c}^{T-c} G_{1}(t, s)\left(\int_{c}^{T-c} G_{2}(s, \tau) g(\tau, u(\tau)) d \tau\right)^{l_{1}} d s \\
& \geq C_{3} \int_{c}^{T-c} G_{1}(t, s)\left(\frac{c^{m-1}}{T^{m-1}} \int_{c}^{T-c} I_{2}(\tau) C_{4}(u(\tau))^{l_{2}} d \tau\right)^{l_{1}} d s \\
& \geq C_{3} C_{4}^{l_{1}} \frac{c^{l_{1}(m-1)}}{T^{l_{1}(m-1)}} \int_{c}^{T-c} G_{1}(t, s) \gamma^{l_{1} l_{2}}\|u\|^{l_{1} l_{2}}\left(\int_{c}^{T-c} I_{2}(\tau) d \tau\right)^{l_{1}} d s \\
& \geq C_{3} C_{4}^{l_{1}} \frac{c^{l_{1}(m-1)+n-1}}{T^{l_{1}(m-1)+n-1}} \gamma^{l_{1} l_{2}}\left(\int_{c}^{T-c} I_{1}(s) d s\right)\left(\int_{c}^{T-c} I_{2}(\tau) d \tau\right)^{l_{1}}\|u\|^{l_{1} l_{2}} \\
& \geq\|u\|^{l_{1} l_{2}} \geq\|u\| .
\end{aligned}
$$


Therefore we obtain

$$
\|\mathcal{A} u\| \geq\|u\|, \quad \forall u \in \partial B_{R} \cap P_{0} .
$$

By (3.10), (3.13) and Theorem $1 \mathrm{i})$, we obtain that $\mathcal{A}$ has a fixed point $u_{1} \in$ $\left(\bar{B}_{R} \backslash B_{\delta_{1}}\right) \cap P_{0}$, that is $\delta_{1}<\left\|u_{1}\right\|<R$. Let $v_{1}(t)=\int_{0}^{T} G_{2}(t, s) g\left(s, u_{1}(s)\right) d s$. Then $\left(u_{1}, v_{1}\right) \in P_{0} \times P_{0}$ is a solution of $(S)-(B C)$. In addition $\left\|v_{1}\right\|>0$. Indeed, if we suppose that $v_{1}(t)=0$ for all $t \in[0, T]$, then by using $(H 2)$ we have $f\left(s, v_{1}(s)\right)=f(s, 0)=0$ for all $s \in[0, T]$. This implies $u_{1}(t)=0$ for all $t \in[0, T]$, which contradicts $\left\|u_{1}\right\|>0$. By using Theorem 1.1 from [11] (see [1]), we obtain $u_{1}(t)>0$ and $v_{1}(t)>0$ for all $t \in(0, T-c]$. The proof of Theorem 2 is completed.

Theorem 3. Assume that $(H 1)$, (H2), (H5) and $(H 6)$ hold. Then the problem $(S)-(B C)$ has at least one positive solution $(u(t), v(t)), t \in[0, T]$.

Proof. We consider the cone $P_{0}$ with $c$ given in $(H 6)$. By $(H 5)$ i) we deduce that there exist $C_{5}>0$ and $C_{6}>0$ such that

$$
q_{1}(x) \leq C_{5} x^{\alpha_{1}}+C_{6}, \quad \forall x \in[0, \infty) .
$$

From (H5) ii), for $\varepsilon_{0}>0, \varepsilon_{0}<\left(2^{\alpha_{1}} C_{5} \alpha \beta^{\alpha_{1}}\right)^{-1 / \alpha_{1}}$, we deduce that there exists $C_{7}>0$ such that

$$
q_{2}(x) \leq \varepsilon_{0} x^{\alpha_{2}}+C_{7}, \quad \forall x \in[0, \infty) .
$$

By using (3.14), (3.15) and (H2), for any $u \in P_{0}$, we obtain

$$
\begin{aligned}
(\mathcal{A} u)(t) \leq & \int_{0}^{T} G_{1}(t, s) p_{1}(s) q_{1}\left(\int_{0}^{T} G_{2}(s, \tau) g(\tau, u(\tau)) d \tau\right) d s \\
\leq & \int_{0}^{T} G_{1}(t, s) p_{1}(s)\left[C_{5}\left(\int_{0}^{T} G_{2}(s, \tau) g(\tau, u(\tau)) d \tau\right)^{\alpha_{1}}+C_{6}\right] d s \\
\leq & C_{5} \int_{0}^{T} G_{1}(t, s) p_{1}(s)\left(\int_{0}^{T} G_{2}(s, \tau) g(\tau, u(\tau)) d \tau\right)^{\alpha_{1}} d s \\
& +C_{6} \int_{0}^{T} I_{1}(s) p_{1}(s) d s \\
\leq & C_{5} \int_{0}^{T} I_{1}(s) p_{1}(s)\left[\int_{0}^{T} G_{2}(s, \tau) p_{2}(\tau)\left(\varepsilon_{0}(u(\tau))^{\alpha_{2}}+C_{7}\right) d \tau\right]^{\alpha_{1}} d s+\alpha C_{6} \\
\leq & C_{5} \int_{0}^{T} I_{1}(s) p_{1}(s) d s\left(\int_{0}^{T} I_{2}(\tau) p_{2}(\tau) d \tau\right)^{\alpha_{1}}\left(\varepsilon_{0}\|u\|^{\alpha_{2}}+C_{7}\right)^{\alpha_{1}}+\alpha C_{6} \\
= & C_{5} \alpha \beta^{\alpha_{1}}\left(\varepsilon_{0}\|u\|^{\alpha_{2}}+C_{7}\right)^{\alpha_{1}}+\alpha C_{6} \\
\leq & 2^{\alpha_{1}} C_{5} \alpha \beta^{\alpha_{1}}\left(\varepsilon_{0}^{\alpha_{1}}\|u\|^{\alpha_{1} \alpha_{2}}+C_{7}^{\alpha_{1}}\right)+\alpha C_{6} \\
= & C_{5} 2^{\alpha_{1}} \varepsilon_{0}^{\alpha_{1}} \alpha \beta^{\alpha_{1}}\|u\|^{\alpha_{1} \alpha_{2}}+C_{5} 2^{\alpha_{1}} \alpha \beta^{\alpha_{1}} C_{7}^{\alpha_{1}}+\alpha C_{6}, \quad \forall t \in[0, T] .
\end{aligned}
$$

By definition of $\varepsilon_{0}$, we can choose sufficiently large $R_{1}>0$ such that

$$
\|\mathcal{A} u\| \leq\|u\|, \quad \forall u \in \partial B_{R_{1}} \cap P_{0} .
$$


From (H6) i), we deduce that there exist positive constants $C_{8}>0$ and $x_{3}>0$ such that $f(t, x) \geq C_{8} x^{\beta_{1}}$, for all $x \in\left[0, x_{3}\right]$ and $t \in[c, T-c]$. From (H6) ii), for $\varepsilon_{1}=\left(\frac{T^{n-1+\beta_{1}(m-1)}}{C_{8} c^{n-1+\beta_{1}(m-1)} \gamma^{\beta_{1} \beta_{2}} \theta_{1} \theta_{2}^{\beta_{1}}}\right)^{1 / \beta_{1}}>0$, we deduce that there exists $x_{4}>0$ such that $g(t, x) \geq \varepsilon_{1} x^{\beta_{2}}$ for all $x \in\left[0, x_{4}\right]$ and $t \in[c, T-c]$.

We consider $x_{5}=\min \left\{x_{3}, x_{4}\right\}$. So we obtain

$$
f(t, x) \geq C_{8} x^{\beta_{1}}, \quad g(t, x) \geq \varepsilon_{1} x^{\beta_{2}}, \forall(t, x) \in[c, T-c] \times\left[0, x_{5}\right] .
$$

From assumption $q_{2}(0)=0$ and the continuity of $q_{2}$, we deduce that there exists sufficiently small $\varepsilon_{2} \in\left(0, \min \left\{x_{5}, 1\right\}\right)$ such that $q_{2}(x) \leq \beta^{-1} x_{5}$ for all $x \in\left[0, \varepsilon_{2}\right]$. Therefore for any $u \in \partial B_{\varepsilon_{2}} \cap P_{0}$ and $s \in[0, T]$, we have

$$
\begin{aligned}
\int_{0}^{T} G_{2}(s, \tau) g(\tau, u(\tau)) d \tau & \leq \int_{0}^{T} G_{2}(s, \tau) p_{2}(\tau) q_{2}(u(\tau)) d \tau \\
& \leq \beta^{-1} x_{5} \int_{0}^{T} I_{2}(\tau) p_{2}(\tau) d \tau=x_{5} .
\end{aligned}
$$

By (3.17), (3.18), Lemma 6 and Lemma 7, for any $t \in[c, T-c]$, we have

$$
\begin{aligned}
(\mathcal{A} u)(t) & \geq \int_{c}^{T-c} G_{1}(t, s) f\left(s, \int_{0}^{T} G_{2}(s, \tau) g(\tau, u(\tau)) d \tau\right) d s \\
& \geq C_{8} \int_{c}^{T-c} G_{1}(t, s)\left(\int_{c}^{T-c} G_{2}(s, \tau) g(\tau, u(\tau)) d \tau\right)^{\beta_{1}} d s \\
& \geq C_{8} \int_{c}^{T-c} G_{1}(t, s)\left(\varepsilon_{1} \int_{c}^{T-c} G_{2}(s, \tau)(u(\tau))^{\beta_{2}} d \tau\right)^{\beta_{1}} d s \\
& \geq \frac{C_{8} c^{n-1}}{T^{n-1}} \int_{c}^{T-c} I_{1}(s)\left[\left(\frac{\varepsilon_{1} c^{m-1}}{T^{m-1}}\right)^{\beta_{1}}\left(\int_{c}^{T-c} I_{2}(\tau)(u(\tau))^{\beta_{2}} d \tau\right)^{\beta_{1}}\right] d s \\
& \geq \frac{C_{8} c^{n-1+(m-1) \beta_{1}} \varepsilon_{1}^{\beta_{1}} \gamma^{\beta_{1} \beta_{2}} \theta_{1} \theta_{2}^{\beta_{1}}}{T^{n-1+(m-1) \beta_{1}}}\|u\|^{\beta_{1} \beta_{2}}=\|u\|^{\beta_{1} \beta_{2}} \geq\|u\| .
\end{aligned}
$$

Therefore

$$
\|\mathcal{A} u\| \geq\|u\|, \quad \forall u \in \partial B_{\varepsilon_{2}} \cap P_{0} .
$$

By (3.16), (3.19) and Theorem 1 ii), we deduce that $\mathcal{A}$ has at least one fixed point $u_{2} \in\left(\bar{B}_{R_{1}} \backslash B_{\varepsilon_{2}}\right) \cap P_{0}$. Then our problem $(S)-(B C)$ has at least one positive solution $\left(u_{2}, v_{2}\right) \in P_{0} \times P_{0}$ where $v_{2}(t)=\int_{0}^{T} G_{2}(t, s) g(s, u(s)) d s$. The proof of Theorem 3 is completed.

\section{Examples}

In this section, we shall present two examples which illustrate our results.

Example 1. Let

$$
f(t, x)=\frac{x^{a}}{t^{\gamma_{1}}(T-t)^{\delta_{1}}}, \quad g(t, x)=\frac{x^{b}}{t^{\gamma_{2}}(T-t)^{\delta_{2}}},
$$


with $a, b>1$ and $\gamma_{1}, \delta_{1}, \gamma_{2}, \delta_{2} \in(0,1)$. Here $f(t, x)=p_{1}(t) q_{1}(x)$ and $g(t, x)=$ $p_{2}(t) q_{2}(x)$, where

$$
p_{1}(t)=\frac{1}{t^{\gamma_{1}}(T-t)^{\delta_{1}}}, \quad p_{2}(t)=\frac{1}{t^{\gamma_{2}}(T-t)^{\delta_{2}}}, \quad q_{1}(x)=x^{a}, \quad q_{2}(x)=x^{b} .
$$

We have $0<\int_{0}^{T} p_{1}(s) d s<\infty, 0<\int_{0}^{T} p_{2}(s) d s<\infty$.

In (H3), for $r_{1}<a, r_{2}<b$ and $r_{1} r_{2} \geq 1$, we have

$$
\limsup _{x \rightarrow 0^{+}} \frac{q_{1}(x)}{x^{r_{1}}}=\lim _{x \rightarrow 0^{+}} x^{a-r_{1}}=0, \quad \limsup _{x \rightarrow 0^{+}} \frac{q_{2}(x)}{x^{r_{2}}}=\lim _{x \rightarrow 0^{+}} x^{b-r_{2}}=0 .
$$

In $(H 4)$, for $l_{1}<a, l_{2}<b, l_{1} l_{2} \geq 1$ and $c \in\left(0, \frac{T}{2}\right)$, we have

$$
\begin{aligned}
& \liminf _{x \rightarrow \infty} \inf _{t \in[c, T-c]} \frac{f(t, x)}{x^{l_{1}}}=\liminf _{x \rightarrow \infty} \inf _{t \in[c, T-c]} \frac{x^{a-l_{1}}}{t^{\gamma_{1}}(T-t)^{\delta_{1}}} \\
& \quad=\left(\max \left\{\frac{\gamma_{1}^{\gamma_{1}} \delta_{1}^{\delta_{1}} T^{\gamma_{1}+\delta_{1}}}{\left(\gamma_{1}+\delta_{1}\right)^{\gamma_{1}+\delta_{1}}}, c^{\gamma_{1}}(T-c)^{\delta_{1}}, c^{\delta_{1}}(T-c)^{\gamma_{1}}\right\}\right)^{-1} \lim _{x \rightarrow \infty} x^{a-l_{1}}=\infty
\end{aligned}
$$

In a similar manner, we have

$$
\liminf _{x \rightarrow \infty} \inf _{t \in[c, T-c]} \frac{g(t, x)}{x^{l_{2}}}=\infty .
$$

For example, if $a=2, b=3 / 2, r_{1}=1, r_{2}=4 / 3, l_{1}=3 / 2, l_{2}=1$, the above conditions are satisfied. Under the assumption $(H 1)$, by Theorem 2 , we deduce that the problem $(S)-(B C)$ has at least one positive solution.

Example 2. Let $f(t, x)=\frac{x^{a}(2+\cos x)}{t^{\gamma}}, g(t, x)=\frac{x^{b}(1+\sin x)}{(T-t)^{\delta}}$, with $a, b \in(0,1)$ and $\gamma, \delta \in(0,1)$. Here $f(t, x)=p_{1}(t) q_{1}(x)$ and $g(t, x)=p_{2}(t) q_{2}(x)$, where

$p_{1}(t)=\frac{1}{t^{\gamma}}, \quad p_{2}(t)=\frac{1}{(T-t)^{\delta}}, \quad q_{1}(x)=x^{a}(2+\cos x), \quad q_{2}(x)=x^{b}(1+\sin x)$.

We have $0<\int_{0}^{T} p_{1}(s) d s<\infty, 0<\int_{0}^{T} p_{2}(s) d s<\infty$.

In $(H 5)$, for $\alpha_{1}=a, \alpha_{2}>b$ and $\alpha_{1} \alpha_{2} \leq 1$, we have

$$
\begin{aligned}
& \limsup _{x \rightarrow \infty} \frac{q_{1}(x)}{x^{\alpha_{1}}}=\limsup _{x \rightarrow \infty} \frac{x^{a}(2+\cos x)}{x^{\alpha_{1}}}=3, \\
& \limsup _{x \rightarrow \infty} \frac{q_{2}(x)}{x^{\alpha_{2}}}=\limsup _{x \rightarrow \infty} \frac{x^{b}(1+\sin x)}{x^{\alpha_{2}}}=0 .
\end{aligned}
$$

In $(H 6)$, for $\beta_{1}=a, \beta_{2}>b, \beta_{1} \beta_{2} \leq 1$ and $c \in\left(0, \frac{T}{2}\right)$, we have

$$
\begin{aligned}
\liminf _{x \rightarrow 0^{+}} \inf _{t \in[c, T-c]} \frac{f(t, x)}{x^{\beta_{1}}} & =\liminf _{x \rightarrow 0^{+}} \inf _{t \in[c, T-c]} \frac{x^{a}(2+\cos x)}{t^{\gamma} x^{\beta_{1}}}=\frac{3}{(T-c)^{\gamma}}>0, \\
\liminf _{x \rightarrow 0^{+}} \inf _{t \in[c, T-c]} \frac{g(t, x)}{x^{\beta_{2}}} & =\liminf _{x \rightarrow 0^{+}} \inf _{t \in[c, T-c]} \frac{x^{b}(1+\sin x)}{(T-t)^{\delta} x^{\beta_{2}}} \\
& =\frac{1}{(T-c)^{\delta}} \lim _{x \rightarrow 0^{+}} x^{b-\beta_{2}}=\infty .
\end{aligned}
$$


For example, if $a=1 / 3, b=1 / 2, \alpha_{1}=1 / 3, \alpha_{2}=1, \beta_{1}=1 / 3, \beta_{2}=1$, the above conditions are satisfied. Under the assumption $(H 1)$, by Theorem 3 , we deduce that the problem $(S)-(B C)$ has at least one positive solution.

\section{Acknowledgement}

The authors thank the referees for their valuable comments and suggestions. The work of the second author was supported by the CNCS grant PN-II-IDPCE-2011-3-0557, Romania.

\section{References}

[1] P.W. Eloe and J. Henderson. Positive solutions for $(n-1,1)$ conjugate boundary value problems. Nonlinear Anal., 28:1669-1680, 1997. http://dx.doi.org/10.1016/0362-546X(95)00238-Q.

[2] D. Franco, G. Infante and D. O'Regan. Nontrivial solutions in abstract cones for Hammerstein integral systems. Dyn. Contin. Discrete Impuls. Syst. Ser. A Math. Anal., 14:837-850, 2007.

[3] C.S. Goodrich. Nonlocal systems of BVPs with asymptotically superlinear boundary conditions. Comment. Math. Univ. Carolin., 53:79-97, 2012.

[4] J. Henderson and R. Luca. Positive solutions for a system of higher-order multipoint boundary value problems. Comput. Math. Appl., 62:3920-3932, 2011. http://dx.doi.org/10.1016/j.camwa.2011.09.045.

[5] J. Henderson and R. Luca. On a system of higher-order multi-point boundary value problems. Electron. J. Qual. Theory Diff. Equ., 2012(49):1-14, 2012.

[6] J. Henderson and R. Luca. Positive solutions for a system of second-order multipoint boundary value problems. Appl. Math. Comput., 218:6083-6094, 2012. http://dx.doi.org/10.1016/j.amc.2011.11.092.

[7] J. Henderson and R. Luca. Positive solutions for singular systems of multi-point boundary value problems. Math. Methods Appl. Sci., 36:814-828, 2013. http://dx.doi.org/10.1002/mma.2628.

[8] G. Infante and P. Pietramala. Eigenvalues and non-negative solutions of a system with nonlocal BCs. Nonlinear Stud., 16:187-196, 2009.

[9] G. Infante and P. Pietramala. Existence and multiplicity of non-negative solutions for systems of perturbed Hammerstein integral equations. Nonlinear Anal., 71:1301-1310, 2009. http://dx.doi.org/10.1016/j.na.2008.11.095.

[10] Y. Ji and Y. Guo. The existence of countably many positive solutions for some nonlinear $n$th order $m$-point boundary value problems. J. Comput. Appl. Math., 232:187-200, 2009. http://dx.doi.org/10.1016/j.cam.2009.05.023.

[11] Y. Ji, Y. Guo and C. Yu. Positive solutions to $(n-1, n) m$-point boundary value problems with dependence on the first order derivative. Appl. Math. Mech. (English Ed.), 30:527-536, 2009.

[12] B. Liu, L. Liu and Y. Wu. Positive solutions for singular systems of threepoint boundary value problems. Comput. Math. Appl., 53:1429-1438, 2007. http://dx.doi.org/10.1016/j.camwa.2006.07.014. 
[13] S. Roman. Linear differential equation with additional conditions and formulae for Green's function. Math. Model. Anal., 16:401-417, 2011. http://dx.doi.org/10.3846/13926292.2011.602125.

[14] H. Su, Z. Wei, X. Zhang and J. Liu. Positive solutions of $n$-order and $m$-order multi-point singular boundary value system. Appl. Math. Comput., 188:12341243, 2007. http://dx.doi.org/10.1016/j.amc.2006.10.077.

[15] Z. Yang. Positive solutions to a system of second-order nonlocal boundary value problems. Nonlinear Anal., 62:1251-1265, 2005.

http://dx.doi.org/10.1016/j.na.2005.04.030.

[16] Y. Zhou and Y. Xu. Positive solutions of three-point boundary value problems for systems of nonlinear second order ordinary differential equations. J. Math. Anal. Appl., 320:578-590, 2006. http://dx.doi.org/10.1016/j.jmaa.2005.07.014. 